\title{
Comprehensive geriatric assessment in nephrology
}

\author{
Ana Farinha ${ }^{1}$, Sofia Duque ${ }^{2}$ \\ 1 Nephrology Department, Centro Hospitalar de Setúbal \\ 2 Orthogeriatric Unit (Coordinator), Internal Medicine Department, Centro Hospitalar de Lisboa Ocidental
}

\section{ABSTRACT}

In Portugal, in $2018,64.5 \%$ of dialysis patients were elderly and nearly a quarter were older than 80 years. Aging is a heterogeneous process: in some patients, there is a marked clinical improvement under renal replacement therapy (RRT) although others show an inexorable functional decline. Despite advances in dialysis, patient mortality rates remain high. These two phenomena have been difficult to understand and it may be difficult to decide which patients may benefit from RRT or conservative care. Comprehensive Geriatric Assessment (CGA) is an instrument used by geriatricians to evaluate functionality and geriatric syndromes, allowing recognition of problems that would not otherwise be identified. It has been used to individualize and tailor therapies. CGA allows frailty identification (the major geriatric syndrome) and might better predict clinical prognosis and interventions' effectiveness. Physical frailty is a multifactorial condition that is common in chronic kidney disease (CKD) patients and its reversibility is hardly predictable when RRT is started so CGA might contribute to a better understanding of frailty in these patients. Therefore, it is possible that CGA application to CKD older patients would contribute to the selection of patients who might benefit the most from RRT and to improve patient clinical condition. This article aims to review geriatric syndromes and frailty in CKD patients, CGA potential to evaluate them and possible models to apply it.

Keywords: aging, chronic kidney disease, conservative care, frailty, geriatric syndrome, renal replacement therapy

\section{INTRODUCTION}

Aging is a dynamic, progressive, universal and irreversible process that is manifested by broadly predictable morphologic and physiologic changes caused by time but influenced by genetics and environment. It leads to a decline in adaptation capacity caused by loss of homeostatic mechanisms of all organs and systems. Consequently, there is less resilience to stressful events (hospitalization, nursing home admission, starting a new drug or treatment, such as dialysis) which translates into greater vulnerability to adverse events and to a greater incidence of pathological issues that will culminate in loss of autonomy, dependence and, finally, death ${ }^{1}$. At a socio demographic level, aging is the result of improved health care (reduction in infant mortality), changes in society (reduction in fertility) and epidemiological transition that have led to an increase in average life expectancy. In Portugal, in 2017, more than a quarter of population was aged over 65 years old ${ }^{2}$. Nephrology shows the same trend: in $2018,64.5 \%$ of dialysis patients were elderly and nearly a quarter were older than 80 years ${ }^{3}$. Despite advances in dialysis, patient mortality rates remain higher than in cancer ${ }^{4}$. In some patients there is a marked clinical improvement under renal replacement therapy (RRT) while others show an inexorable functional decline. These two patterns of evolution reflect the heterogeneity of aging in Nephrology and in clinical practice it is difficult to predict which trajectory older patients will follow when starting RRT. Therefore, decision to initiate RRT versus conservative care is one of hardest that Nephrologists can face and tools to help in this decision are fundamental. The Comprehensive Geriatric Assessment (CGA) is one of the most important instruments used by geriatricians and their teams to globally assess older patients and plan effective interventions. CGA is a multidimensional, interdisciplinary diagnostic process to determine the medical, cognitive, psychological and functional capabilities of older persons in order to develop a coordinated and integrated plan of treatment and long-term followup, focused on maintenance of autonomy and improvement of quality of life. CGA allows a holistic assessment of older patients and the recognition of geriatric syndromes or other problems that otherwise would not be identified ${ }^{5}$. It has been used in some specialties with the intent to individualize and tailor therapies. It is possible that its application to chronic kidney disease (CKD) patients would improve patient clinical condition and enables the selection of patients who might benefit the most from RRT versus a conservative approach. For both groups, and even in the earlier stages of CKD, CGA can be useful to provide a complete intervention plan and optimize quality of life, autonomy and prognosis. In this article, geriatric syndromes and frailty in CKD patients, the CGA potential to evaluate them and possible models to apply it will be reviewed.

\section{CHRONIC KIDNEY DISEASE AS AN AGING ACCELERATOR}

Chronic kidney disease is associated with premature aging. Some theories have been developed to explain this phenomenon. One involves systemic low-grade inflammation caused by persistent "uremic inflammation" that resembles the one observed in the general population aging process ("inflammaging"), but which happens independent of patient age ${ }^{6}$. Other uses the Klotho protein as a link between these two entities. Klotho has been described as an antiaging protein with 
a central role in cellular senescence where Klotho-deficient mice manifested a syndrome resembling accelerated human aging with extensive and accelerated arteriosclerosis while its over expression would extend average mice life span ${ }^{7}$. For Nephrologists, Klotho is familiar as an inhibitor of vascular calcification that decreases as CKD progresses, in parallel with phosphate accumulation. On an organ/ system level, as a whole, Klotho decrease is linked to a variety of changes, such as endothelial dysfunction, increased vascular stiffness, left ventricular diastolic dysfunction, osteoporosis, cognitive dysfunction, muscular atrophy and malnutrition ${ }^{8}$. All these aspects contribute to unsuccessful aging, a hallmark of CKD.

\section{UNSUCCESSFUL AGING AND GERIATRIC SYNDROMES IN CKD}

Unsuccessful aging may be patent in clinical conditions commonly found in older adults called geriatric syndromes. They share underlying contributing factors and involve multiple organ systems, but they do not fit into specific disease categories (Figure 1). They contribute to poor health outcomes, including disability, institutionalization, dependence and even mortality. They also have a significant impact in quality of life, but they remain frequently undiagnosed. Classic geriatric syndromes, defined by Isaacs in 1970 as Geriatric Giants, include urinary and fecal incontinence, cognitive impairment, instability/falls/immobility and iatrogenesis (pharmacological and non-pharmacological) ${ }^{9}$. Frailty was described more recently but it is considered the major geriatric syndrome and the main focus of Geriatric Medicine. These conditions are more common in CKD patients, even at younger ages and some studies have reported end stage renal disease (ESRD) patients to have a mean of 5.9 geriatric conditions with $98.0 \%$ to have at least one or more ${ }^{10}$.

\section{Cognitive impairment}

Cognitive impairment rises with age as does the decline in renal function ${ }^{11}$. In ESRD patients it is three to four times more common ${ }^{12}$. In the general population, Alzheimer's disease is the most frequent form of dementia. In Portugal, vascular cognitive impairment seems to be more prevalent than Alzheimer's disease, probably due to vascular disease and stroke incidence ${ }^{13}$. In CKD patients, vascular dementia is also more common because of many shared risk factors (albuminuria, anemia, intradialytic hypotension, dialysis disequilibrium, etc) that may impair cerebral perfusion and oxygen supply to brain tissue ${ }^{14}$.

Cognitive impaired older patients may not fully understand the importance of the CKD, its evolution pattern and available therapies. Executive dysfunction may also impair patient compliance with medication, with dietary counseling or taking their own decisions over future care or treatment options (RRT vs conservative care) and its implications. Cognitively impaired patients may also misunderstand the goals of RRT, its indefinite duration and so build false expectations

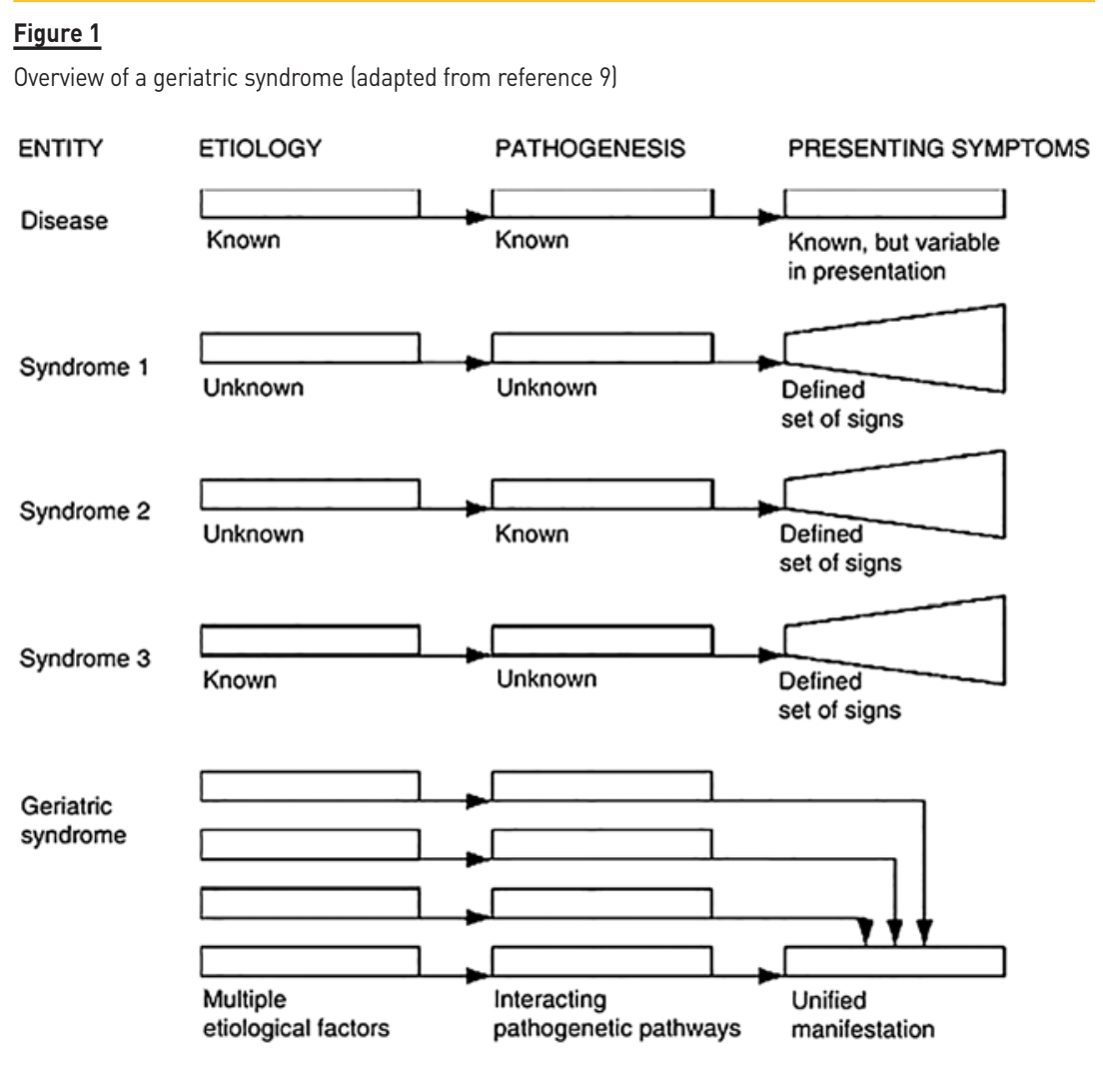


that undermine their quality of life, their wellbeing and, lastly their adherence to RRT. So it is fundamental to diagnose and determine the severity of cognitive impairment in early stages of CKD disease ${ }^{15}$.

Differential diagnosis of cognitive impairment may be more difficult in CKD patients because of multiple situations that are frequently overlapped, such as depression or uremic syndrome. Depression is common in the elderly and is also more prevalent in CKD patients ${ }^{12}$. Fatigue, anorexia or loss of energy are all common to these three entities. Pseudodementia has been used to describe cognitive impairment due to depression that reverts with antidepressant drugs, so these treatment may be initiated when depression is suspected ${ }^{14}$. In the late stages of CKD, cognitive impairment may also be difficult to distinguish from uremic syndrome: uremic toxins accumulation may be responsible for some of central neurological symptoms such as disorientation, lethargy, fatigue, anorexia or sleep disturbances that mimic primary cognitive impairment ${ }^{12}$. Given the reversibility of the uremic syndrome once starting RRT, cognition assessment must be careful at this stage and a diagnosis of dementia should ideally not be considered at the late stage of CKD, unless cognitive impairment clearly started much before the significant rise of uremia. Neuropsychological evaluation may be immediately used in all these differential diagnoses. The most popular screening tool used to detect cognitive impairment, the Mini-Mental State Exam, may not be sensitive enough to identify executive dysfunction in CKD patients, which may cause this condition to go unnoticed or diagnosed late. Other options such as MOCA or the clock drawing task should be preferred ${ }^{12,14}$. Anyway, cognitive impairment is common and its diagnosis and interventions are relevant both for patients' and Nephrologists' decisions.

\section{Instability, falls and immobility}

CKD affects equilibrium, posture and gait by its expression in several systems: disturbing hydro electrolytic balance, cardiovascular system (vascular stiffness, postural hypotension, arrhythmias) central and peripheral nervous system (encephalopathy, peripheral and autonomic neuropathy), musculoskeletal system (myopathy and renal osteodystrophy), nutritional status (metabolic acidosis and malnutrition) or hematological system (anemia). These systems are all deeply related to maintenance of gait ability and minor impairments can lead to postural instability, falls and immobility ${ }^{16}$. In addition, depression and anxiety are also prevalent psychological conditions in older CKD patients that can contribute to immobility ${ }^{11}$. Sarcopenia (muscle failure) is a geriatric condition (previously considered as a geriatric syndrome) that is very frequent in CKD patients and also contributes to increased risk of falls and fractures, impairment on the ability to perform activities of daily living, mobility disorders, loss of independence or need for long-term care placement, poorer quality of life, death and higher healthcare costs. As knowledge in this area has increased, sarcopenia was classified as a new disease and included in the ICD$10^{17}$. Its definition has been evolving and it is considered by many authors as the biological substrate for physical frailty ${ }^{18}$. Sarcopenia is a progressive and generalized skeletal muscle disorder that is characterized by reduction of skeletal muscle mass, muscle strength and physical performance ${ }^{17}$. Primary sarcopenia is a condition purely related to aging muscle loss. Secondary sarcopenia has been described in association to disease, inactivity and malnutrition ${ }^{19}$. CKD has been identified as a possible cause of sarcopenia although older CKD patients will probably present multifactorial aetiology (aging, CKD, inactivity and malnutrition). Prevalence of sarcopenia in CKD is high, increases with age and severity of the disease. Several physiopathological models of sarcopenia in CKD have been suggested but protein intake restriction probably plays an important role $\mathrm{e}^{20}$.

Other aspects that contribute to instability are hearing and vision impairment, which are also more common in both elderly and dialysis patients. A common cause of vision impairment in older patients is retinopathy due to hypertension and diabetes, two common diseases in CKD, but in hemodialysis patients' shifts in volume, blood pressure and electrolytes may also contribute to its occurrence. ${ }^{12}$

Falls are the final consequence of all these contributors. Falls are an important complication in older patients where minor trauma can result in high morbidity fractures, such as hip fracture. Even when there is no fracture, soft tissues lesions can impair balance and gait ability. After a fall, older persons can develop "fear of falling", an underdiagnosed psychological condition that can lead to immobility ${ }^{16}$. Its management is a challenge, based on physical rehabilitation and cognitive behavioral intervention that is not always available. Dialysis patients have a four times higher risk of fall than age-matched nondialysis patients ${ }^{16}$. When a fall happens in a CKD patient, it represents longer hospitalizations, increased risk of institutionalization and increased risk of mortality ${ }^{11}$

When a fall is complicated with fracture, osteoporosis must be considered, if not previously diagnosed. According to WHO, occurrence of a minimal trauma fracture of any bone is enough to establish the diagnosis of osteoporosis. In CKD patients, nevertheless, osteoporosis diagnosis and treatment may be more complex. Evidence of CKDmineral and bone disorder must be ruled out because of overlap between age-related osteoporosis and renal osteodystrophy. Bone biopsy can help in differential diagnosis and treatment, but it is not always feasible. Once osteoporosis diagnosis is considered, pharmacological treatment is also a challenge. Osteoporosis management in CKD patients has been linked to atherosclerosis progression, vascular calcification, and bone turnover suppression, so in stages 4 to 5 , bisphosphonates should not be the first choice. The effect of new antiresorptive agents like denosumab still lacks strong evidence ${ }^{21}$.

So, as CKD progresses, it may be even more difficult to distinguish renal disease's contribution to these geriatric syndromes that are interrelated and that may arise in sequence, in a vicious circle that jeopardizes quality of life and increases mortality. Assessment and prevention of falls should be made in every older person and interventions are even more relevant in older CKD patients considering the worse outcomes.

\section{latrogenesis and polypharmacy}

latrogenesis refers to any effect on a person, resulting from any activity of healthcare professionals, which does not support a goal of the person affected. latrogenesis, which is a disease recognized by WHO, includes adverse unforeseen outcomes resulting from a medication or other non-pharmacological interventions but also from the 
omission of interventions that, according to evidence-based medicine, could benefit the patient.

Both elderly and CKD patients are prone to iatrogenesis because of the decline of homeostatic mechanisms, multimorbidity and higher exposure to medical interventions. Indeed, polypharmacy, usually described as intake of 5 or more drugs or any potentially inappropriate medication, increases the risk of adverse drug reactions. On average, dialysis patients take 10-12 daily prescribed and over-the-counter drugs ${ }^{22}$. However, polypharmacy may be needed to treat properly an older CKD patient. In addition to multiple comorbidities, the possibility of drug-drug or drug-disease interactions is much more worrisome in CKD patients because many drugs are eliminated by kidneys. Adverse drug reactions may lead to two interrelated phenomena: "the prescribing cascade" and "the iatrogenesis cascade". The "prescribing cascade" is a situation where an adverse event is incorrectly interpreted as a new medical condition that requires a new prescription. A classical example is represented in Figure $2^{1}$. Therefore, whenever a new condition is detected, a therapeutic review should be made to identify and correct pharmacological iatrogenesis. Indeed, given the high risk of pharmacological iatrogenesis in CKD older patients, their drug regimen should be periodically reviewed, to identify possible prescribing cascades, drugs not clinically indicated and possible drug interactions. The concept of "potentially inappropriate medication" (PIM) was coined to identify any drug that is not clinically recommended or is risky when safer alternatives are available. To avoid iatrogenesis, lists of PIMs have been published, such as Beers Criteria list and STOPP (Screening Tool of Older Persons' Prescriptions) and START (screening tool to alert doctors to the right treatment) criteria. Both can be used as instruments to guide deprescription. Additionally, STOPP and START include a section to avoid therapeutic nihilism, listing drugs that are frequently omitted despite recognized evidence $^{22}$.

\section{Frailty}

In 2001, Fried and colleagues introduced the term Frailty as a syndrome of decreased physiological reserve and resistance to stressors resulting in an increased vulnerability to adverse outcomes such as functional dependency, hospitalization and mortality. Frailty is now considered the major geriatric syndrome. Underlying mechanisms of frailty include biological aging driven by genetic and environmental factors, unhealthy lifestyle, chronic diseases, malnutrition, inflammation and sarcopenia. By definition, frailty can potentially be prevented or treated with specific modalities, and should not be considered an irreversible condition. Advanced kidney disease involves many pathophysiological changes, such as malnutrition, inflammation and sarcopenia that can contribute to frailty and its higher prevalence even in younger ESRD adults. Thus, CKD is considered a risk factor for frailty ${ }^{23}$. On the other hand, frailty may contribute to worse outcomes in dialysis patients which take us into a vicious cycle. So, identifying frailty may be very important to plan care.

\section{Frailty has been conceptualized in two models:}

- the physical model (Fried model), where five criteria are established to create the frailty phenotype: weight loss, gait speed, weakness (measured by grip strength), decreased physical activity (in Kcal expend per week) and exhaustion (patient response). It is based in pathophysiological features that allow a more objective evaluation but requires patient participation and specialized equipment ${ }^{24}$; this model is not easy to apply in clinical settings and it is mainly used in research;

- the deficit accumulation model (Rockwood model), that is based on the accumulation of several deficits, including illnesses, geriatric syndromes, symptoms, and social problems ${ }^{25}$, from a list of 70 items (the CSHA Frailty Index); although, the CSHA Frailty Index is more easy to apply in clinical practice than the Fried criteria, later a simplified but more subjective version was published to be used in clinical office (the Clinical Frailty Scale - Table 1$)^{28}$.

The Fried model compasses a purely physical approach of frailty; however, in real life, older patients may be physical robust but vulnerable at cognitive, psychological or social dimensions or vice versa; the Rockwood model is a more multidimensional approach than the Fried criteria, considering all the dimensions that can be affected.

\section{Figure 2}

latrogenic cascade. NSAI - non-steroid anti-inflammatory (modified from reference 1).

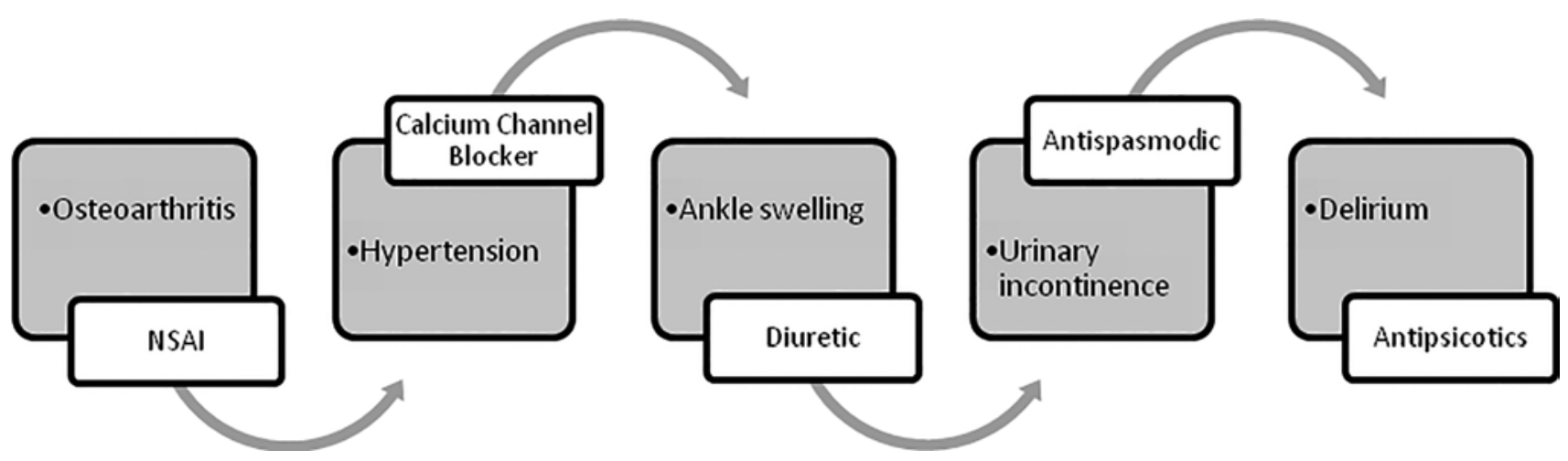


Table 1

Clinical Frailty Scale (adapted from reference 28)

\begin{tabular}{|c|c|}
\hline Evaluation & Description \\
\hline 1. Very Fit & People who are robust, active, energetic and motivated. These people commonly exercise regularly. They are among the fittest for their age. \\
\hline 2. Well & People who have no active disease symptoms but are less fit than category 1. Often, they exercise or are very active occasionally, e,g. seasonally. \\
\hline 3. Managing Well & People whose medical problems are well controlled, but are not regularly active beyond routine walking. \\
\hline 4. Vulnerable & While not dependent on others for daily help, often symptoms limit activities. A common complaint is being "slowed up", and / or being tired during the day. \\
\hline 5. Mildly Frail & $\begin{array}{l}\text { These people often have more evident slowing, and need help in high order IADLs (finances, transportation, heavy housework, medications), Typically, mild } \\
\text { frailty progressively impairs shopping and walking outside alone, meal preparation and housework. }\end{array}$ \\
\hline 6. Moderately Frail & $\begin{array}{l}\text { People need help with all outside activities and with keeping house. Inside, they often have problems with stairs and need help with bathing and might } \\
\text { need minimal assistance (cuing, standby) with dressing, }\end{array}$ \\
\hline 7. Severely Frail & Completely dependent for personal care, from whatever cause (physical or cognitive). Even so, they seem stable and not at high risk of dying (within -6 months). \\
\hline 8. Very Severely Frail & Completely dependent, approaching the end of life. Typically, they could not recover even from a minor illness. \\
\hline 9. Terminally III & Approaching the end of life. \\
\hline \multicolumn{2}{|c|}{ Scoring frailty in people with dementia } \\
\hline \multicolumn{2}{|c|}{ The degree of frailty corresponds to the degree of dementia. } \\
\hline \multicolumn{2}{|c|}{$\begin{array}{l}\text { - Common symptoms in mild dementia include forgetting the details of a recent event, though still remembering the event itself; repeating the same question/story and social withdrawal. } \\
\text { - In moderate dementia, recent memory is very impaired, even though they seemingly can remember their past life events well. They can do personal care with prompting. } \\
\text { - In severe dementia, they cannot do personal care without help. }\end{array}$} \\
\hline
\end{tabular}

Several studies have compared different tools to evaluate frailty in CKD patients ${ }^{26,27}$. Despite the lack of agreement on frailty definition and the challenge of its measurement, it is consensual that it is clinically useful to stratify patients in 3 categories "healthy/usual", "vulnerable" and "frail". RRT decisions should be based on this stratification instead of chronological age. Frail patients have an increased hazard ratio for mortality within their first year of dialysis compared with individuals who did not meet these criteria, after adjustment for multiple factors, including age. That makes frailty into a more sensitive predictor of death than age. Another argument to use this classification is the fact that frailty was associated with earlier initiation of dialysis ${ }^{28}$. Initiation of dialysis at a higher estimated glomerular filtration rate (eGFR) has been associated with poor outcomes in elderly ESRD patients. On the other hand, the association between early initiation and mortality became statistically nonsignificant when frailty was included, suggesting that frailty may be a contributing factor. Another bias that could have confounded this association is the cause of early initiation. Self-reported symptoms of frailty may have been perceived as uremic symptoms, resulting in earlier referral for dialysis initiation at higher eGFR ${ }^{28}$.

\section{COMPREHENSIVE GERIATRIC ASSESSMENT}

Comprehensive Geriatric Assessment (CGA) was first developed in the 1960 s to address the wide heterogeneity in aging. This multidimensional evaluation provides the best estimate of a physiologic reserve to improve functionality, quality of life and to plan individually tailoring interventions and care. CGA has been widely used in geriatric setting for longtime and has been tried outside this specialization. In Oncology, the employment of CGA is gaining interest to choose anti-cancer therapies $^{29}$. In Anesthesiology to a more complete preoperative evaluation of elderly patients has been applied $(29,30)$. In Cardiology there is also a growing awareness of this complementary approach to reach a more integrative cardiovascular care ${ }^{31}$. In Nephrology, CGA could be equally useful in several phases of the disease $\mathrm{s}^{32}$. It could be used

- in earlier stages of CKD (even when progression is not expected) as a geriatric tool used to improve diagnosis, prevent geriatric syndromes, ameliorate discharge trajectories, and increase the chance to avoid institutionalization, in a logic of optimizing the patient's potential;

- in ESRD patients, to identify older adults who are on the progressive trajectory of functional or cognitive decline, to identify additional geriatric syndromes with impact on outcomes, in order to choose the best treatment option (RRT versus conservative care);

- in patients under RRT to manage complexity, to follow quality of life or changes in prognosis or life expectancy that highlight the need to RRT change, assistance or even conservative care.

CGA might also be explored for benchmarking purposes.

The standard CGA is performed by a multidisciplinary team (geriatrician doctor and nurse, nutritionist, social worker, occupational therapist,...) that conducts an inclusive multidimensional evaluation. The essential dimensions include functional status, cognition, comorbidities, mood, falls, polypharmacy, nutrition, social support and financial wellbeing. The evaluation can be extended to caregiver burden, spirituality, sensory impairments, goals of care and advance care planning ${ }^{32}$. At first, these dimensions were evaluated separately with specific instruments, but further research has compiled single approaches in complex, multidimensional tools that have been validated in several clinical contexts inclusive with prognostic intentions (Multidimensional Prognostic Index, Edmonton Frailty Scale,...) (Tables 2 and 3) ) $^{34,35}$.

The usefulness of a geriatric assessment has been proposed for several nephrology associations. European Renal Association in its recent European Best Practice Guidelines on Management of Older 


\section{Table 2}

Edmonton Frailty Scale ladapted from reference 9).

\begin{tabular}{|c|c|c|c|c|}
\hline Frailty Domain & Item & 0 point & 1 point & 2 points \\
\hline Cognition & $\begin{array}{l}\text { Please imagine that this pre-drawn circle is a clock. I would like you to place the numbers in } \\
\text { the correct positions then place the hands to indicate a time of 'ten after eleven' }\end{array}$ & No errors & $\begin{array}{l}\text { Minor spacing } \\
\text { errors }\end{array}$ & Other errors \\
\hline \multirow[t]{2}{*}{ General health status } & In the past year, how many times have you been admitted to a hospital? & 0 & $1-2$ & $\geq 2$ \\
\hline & In general, how would you describe your health? & $\begin{array}{l}\text { 'Excellent', 'Very } \\
\text { good', 'Good' }\end{array}$ & 'Fair' & 'Poor' \\
\hline Functional independence & $\begin{array}{l}\text { With how many of the following activities do you require help? (meal preparation, shopping, } \\
\text { transportation, telephone, housekeeping, laundry, managing money, taking medications) }\end{array}$ & $0-1$ & $2-4$ & $5-8$ \\
\hline Social support & When you need help, can you count on someone who is willing and able to meet your needs? & Always & Sometimes & Never \\
\hline \multirow[t]{2}{*}{ Medication use } & Do you use five or more different prescription medications on a regular basis? & No & Yes & \\
\hline & At times, do you forget to take your prescription medications? & No & Yes & \\
\hline Nutrition & Have you recently lost weight such that your clothing has become looser? & No & Yes & \\
\hline Mood & Do you often feel sad or depressed? & No & Yes & \\
\hline Continence & Do you have a problem with losing control of urine when you don't want to? & No & Yes & \\
\hline Functional performance & $\begin{array}{l}\text { I would like you to sit in this chair with your back and arms resting. Then, when I say 'GO', } \\
\text { please stand up and walk at a safe and comfortable pace to the mark on the floor } \\
\text { (approximately } 3 \text { m away), return to the chair and sit down' }\end{array}$ & $0-10 s$ & $11-20 \mathrm{~s}$ & $\begin{array}{c}\text { One of }>20 \mathrm{~s} \\
\text { patient unwilling, } \\
\text { or requires } \\
\text { assistance }\end{array}$ \\
\hline Totals & Final score is the sum of column totals & & & \\
\hline
\end{tabular}

\section{Table 3}

Multidimensional Prognostic Index (adapted from reference 34).

\begin{tabular}{|c|c|c|c|}
\hline & & Problem severity & \\
\hline & No (=0 points) & Minor (=0.5points) & Severe (=1point) \\
\hline 1. Co-habitation status & Living with family & Institutionalized & Living alone \\
\hline 2. Current medication use & $0-3$ & $4-6$ & $\geq 7$ \\
\hline $\begin{array}{l}\text { 3. Activities of daily living (ADL) score } \\
\text { Number of active functional activities }\end{array}$ & $6-5$ & $4-3$ & $2-0$ \\
\hline $\begin{array}{l}\text { 4. Instrumental activities of daily living scale (IADL) score } \\
\text { Number of active functional activities }\end{array}$ & $8-6$ & $5-4$ & $3-0$ \\
\hline $\begin{array}{l}\text { 5. Short portable mental status } \\
\text { Questionnaire (SPMSQ) score }\end{array}$ & $0-3$ & $4-7$ & $8-10$ \\
\hline $\begin{array}{l}\text { 6. Cumulative illness rating scale (CIRS) } \\
\text { Number of diseases }\end{array}$ & 0 & $1-2$ & $\geq 3$ \\
\hline 7. Mini nutritional assessment (MNA) & $\geq 24$ satisfactory nutritional status & $17-23.5$ at risk of malnutrition & $<17$ malnutrition \\
\hline 8. Exton-Smith scale (ESS) & $\begin{array}{c}16-20 \\
\text { minimum risk }\end{array}$ & $\begin{array}{c}10-15 \\
\text { Moderate risk }\end{array}$ & $\begin{array}{l}5-9 \\
\text { High risk }\end{array}$ \\
\hline $\begin{array}{l}\text { Total of MPI score (sum of } 8 \text { scales } / 8 \text { ) } \\
- \text { Low risk }<0.33 \\
\text { - Moderate risk } 0.34-0.66 \\
\text { - High risk }>0.66\end{array}$ & & & \\
\hline
\end{tabular}

patients with CKD proposes some measures and pathways to handle elderly patients with decreased renal function. It includes evaluation of renal function and risk of progression, but also highlights the importance of assessing frailty, functional decline and malnutrition to plan care in this specific population ${ }^{36}$. From the KDIGO group, the Dialysis initiation, modality choice, access and prescription Controversies Conference underlines, as a key question, the need to define frailty indexes in decision-making ${ }^{37}$. The National Kidney Foundation stresses the value of a person-centered care instead of a disease-based approach in older adults with kidney disease ${ }^{38}$. Even International Society of Peritoneal Dialysis made a review on elderly peritoneal dialysis patients focusing geriatric assessment issues ${ }^{39}$. At an individual level, Nephrologists also feel and express their limitations in geriatric approach and the need to deepen knowledge in this field ${ }^{40}$. 
Although its importance is recognized, the means to implement CGA in Nephrology units have not been established and require special skills. Several models have been studied: a geriatrician making part of the staff, nephrologists receiving additional training in geriatrics to perform these evaluations ${ }^{41}$, a geriatric nephrology nurse conducting interviews ${ }^{42}$ or self-reported questionnaires answered by patients and caregivers. The most relevant reasons why there is no single way to perform CGA in Nephrology units are the barriers that have been identified. Resources needed are one of them. They include time, space, and personnel to conduct CGA, available geriatric expertise, and infrastructure for care coordination and implementation of care plans. Another concern is cost, even if cost-effectiveness is yet unclear because of heterogeneity of approach across studies. Anyway at a patient and caregiver level, CGA was thought to help to deal with problems more adequately ${ }^{10}$.

Recognizing these barriers, Brown et al. have structured a feasibility project to determine whether a nephrology nurse could deliver a modified geriatric assessment (MGA. The MGA includes assessment of dependence on mobility aids, falls, presence of vision or hearing problems, and social support provided by family or other caregivers, as well as assessment of frailty and cognitive dysfunction ${ }^{42}$. It takes up to an hour which means that it could be completed while waiting for hemodialysis or in peritoneal dialysis clinic either as a whole or in smaller components on separate occasions. Preliminary results have been encouraging. Other projects of the kind have been implemented worldwide. The POLDER (Pathway for Older Patients Reaching End Stage Renal Disease) initiative is a pilot project from multiple Dutch hospitals and academic centers that will work together to study the implementation feasibility of a geriatric assessment in routine pre-dialysis care. It will evaluate clinical and geriatric data in a national database but results have not been published yet ${ }^{43}$. These groundbreaking projects along with the recognition that the way we currently manage our patients is not enough will surely bring new awareness and interests in this area, which, we hope, will improve the care provided to our senior patients.

\section{CONCLUSION}

As long as the older CKD population continues to grow but outcomes continue to worsen compared to the general population, collaboration between nephrologists and geriatricians might aid us in improving medical care for older patients with kidney disease. Since Geriatric Medicine is still not widely available in the Portuguese national health system, a knowledge of geriatrics is important for the integration of these issues in clinical practice. Some authors defend that morbidity and mortality derive more from the presence of geriatric syndromes than from the diseases that cause them ${ }^{9}$. In fact, there is no doubt that geriatric syndromes predispose elderly ESRD patients to adverse outcomes, so it should trigger a careful consideration of which kind of care might be appropriate. Thus, it may be the time to look at CKD patients from another perspective: that CGA may be the starting point for an improvement in outcomes that we have not yet attained.

Disclosure of potential conflicts of interest: none declared

\section{Acknowledgements}

To Prof. Ivan Carlos Ferreira Antonello, MD, Phd, Division of Nephrology, Department of Internal Medicine, Medicine School, Pontifícia Universidade Católica do Rio Grande do Sul, Porto Alegre, Brazil for the first revision and orientation

\section{References}

1. Elizabete Viana de Freitas, Ligia Py. Estudo da Velhice: Histórico, definição do Campo e termos básicos in Tratado de Geriatria e Gerentologia. 4th Edition. Guanabara Koogan, 2017: 1-13

2. Portdata. Contemporary Portugal Database. Available at https://www.pordata.pt/Portugal Accessed Jun, 2019

3. Replacement Renal Therapy of Chronic Renal Disease in Portugal (2017) Available at http:www spnefro.pt/comissoes_Gabinete_registo_2017/registo_2017. Accessed Jun, 2019

4. Farinha A. Prognostication in End-Stage Renal Disease. Port J Nephrol Hypert 2016; 30(4): 246-251

5. Elizabete Viana de Freitas, Ligia Py. Avaliação Geriátrica Ampla in Tratado de Geriatria e Gerentologia. 4th Edition. Guanabara Koogan, 2017: 152-167

6. Kooman JP, Dekker MJ, Usvyat LA, P Kotanko, van der Sande FM, Schalkwijk CG et al. Inflammation and premature aging in advanced chronic kidney disease Am J Physiol Renal Physiol 2017;313:F938F950

7. Zou D, Wu W, He Y, Ma S, Gao J. The role of klotho in chronic kidney disease. BMC Nephrol. 2018: 22;19(1):285

8. Hu MC, Kuro-o M, Moe OW. Klotho and chronic kidney disease. Contrib Nephrol. 2013; 180: 47-63

9. Veríssimo MT. Síndromes Geriátricas in Geriatria Fundamental. Lidel. 2014: 353-358

10. Parlevliet JL, Buurman BM, Pannekeet MM, Boeschoten EM, ten Brinke L, Hamaker ME et al. Systematic comprehensive geriatric assessment in elderly patients on chronic dialysis: a cross-sectional comparative and feasibility study. BMC Nephrol. 2012; 30:13:30

11. Bhattarai M. Geriatric Issues in older dialysis patients. R I Med J. 2016;1;99(7):15-8.

12. Wiggins J, Bitzer M. Geriatric assessment for the nephrologist. Semin Dial. 2012;25(6):623-627

13. Ruano L, Araújo N, Branco M, Barreto R, Moreira S, Pais R et al. Prevalence and causes of cognitive impairment and dementia in a population-based cohort From northern Porugal. Am J Alzheimers Dis Other Demen. 2019 Feb;34(1):49-56

14. Tamura MK, Recognizing delirium, dementia and depression in Geriatric Nephrology Curriculum Available at https://www.asn-online.org/education/distancelearning/curricula/geriatrics/Chapter36.pdf. Accessed Jul, 2019

15. Abdulla A, Wright PN, Ross LE, Gallagher H, lyasere O, Ma N et al. Proceedings From the Symposium on Kidney Disease in Older People: Royal Society of Medicine, London, January 19 2017. Gerontol Geriatr Med. eCollection 2017 Dec 7;3:2333721417736858

16. Morley EJ. Falls in Elderly Patients With Kidney Disease in Geriatric Nephrology Curriculum Available at https://www.asn-online.org/education/distancelearning/curricula/geriatrics/Chapter33.pdf. Accessed Jul, 2019

17. Anker SD, Morley JE, von Haehling S, Welcome to the ICD-10 code for sarcopenia. J Cachexia Sarcopenia Muscle. 2016;7(5):512-514

18. Bernabei R, Martone AM, Vetrano DL, Calvani R, Landi F, Marzetti E. Frailty, physical frailty, sarcopenia: A new conceptual model. Stud Health Technol Inform. 2014;203:78-84

19. Cruz-Jentoft JC, Bahat G, Bauer J, Boirie $Y$, Bruyère $O$, Cederholm T, et al. Sarcopenia: Revised European consensus on definition and diagnosis. Age Ageing. 2019;48(1):16-31

20. D'Alessandro C, Piccoli GB, Barsotti M, Tassi S, Giannese D,Morganti R, Cupisti A. Prevalence and correlates of sarcopenia among elderly CKD putpatients on tertiary care. Nutrients. 2018; 10(12): 1951

21. Miller PD. Osteoporosis in patients with chronic kidney disease: Management. Available at Uptodate. Accessed Jul, 2019

22. Alagiakrishnan K, Mah D, Padwal R. Clinical challenges and emerging approaches to medication therapy in older adults. Discov Med. 2018;26(143):137-146

23. Abdel-Rahman E. Association Between CKD and Frailty and Prevention of Functional Losses in Geriatric Nephrology Curriculum Available at https://www.asn-online.org/education/ distancelearning/curricula/geriatrics/Chapter34.pdf. Accessed Jul, 2019

24. Fried LP, Tangen CM, Walston J et al. Cardiovascular Health Study Collaborative Research Group: Frailty in older adults: evidence for a phenotype. J Gerontol A Biol Sci Med Sci. 2001:56:M146-156

25. Rockwood K, Song X, MacKnight C et al. A global clinical measure of fitness and frailty in elderly people. CMAJ. 2005; 173:489-495

26. van Loon IN, Goto NA, Boereboom FTJ, Bots ML, Verhaar MC, Hamaker ME. Frailty screening tools for elderly patients incident to dialysis. Clin J Am Soc Nephrol. 2017;12(9):1480-1488

27. Chowdhury R, Peel NM, Krosch M, Hubbard RE. Frailty and chronic kidney disease: A systematic review. Arch Gerontol Geriatr. 2017;68:135-142

28. Musso CG, Jauregui JR, Macías Núñez JF. Frailty phenotype and chronic kidney disease: A review of the literature. Int Urol Nephrol. 2015;47(11):1801-1807

29. Maas HA, Janssen-Heijnen ML, Olde Rikkert MG, Machteld Wymenga AN. Comprehensive geriatric assessment and its clinical impact in oncology. Eur J Cancer. 2007:43(15):2161-2169

30. Eamer G, Taheri A, Chen SS, Daviduck $Q$, Chambers T, Shi X, Khadaroo RG. Comprehensive geriatric assessment for older people admitted to a surgical service. Cochrane Database Syst Rev 1: CD012485, 2018

31. Byerly LK, Harper GM. Is it time for comprehensive geriatric assessment to move beyond primary care? The case for targeting medical sub-specialty practice. Isr J Health Policy Res. 2017;6:33

32. Gambert SR. Comprehensive Geriatric Assessment: A Multidimensional Process Designed to Assess an Elderly Person's Functional Ability, Physical Health, Cognitive and Mental Health, and SocioEnvironmental in Geriatric Nephrology Curriculum Available at https://www.asn-online.org/ education/distancelearning/curricula/geriatrics/Chapter26.pdf. Accessed Jul, 2019

33. Hall RK, McAdams-DeMarco MA. Breaking the cycle of functional decline in older dialysis patients. Semin Dial. 2018;31(5):462-467

34. Rengo F, Parisi V, Rengo G, Femminella GD, Rengo C, Zincarelli $C$ et al. Instruments for geriatric assessment: new multidimensional assessment approaches. J Nephrol. 2012;25(19): $573-78$ 
35. Orlandi F, GesualdoGD. Assessment of the frailty level of elderly people with chronic kidney disease undergoing hemodialysis. Acta Paul Enferm. 2014; 27(1):29-34

36. Farrington K, Covic A, Aucella F, Clyne N, de Vos L, Findlay A et al. ERBP Guideline Development Group: Clinical Practice Guideline on management of older patients with chronic kidney disease stage 3b or higher (eGFR,45 mL/min/1.73 m2). Nephrol Dial Transplant.2016;31[suppl 2]: ii1-ii66

37. Chan CT, Blankestijn PJ, Dember LM, Gallieni M, Harris DCH, Lok CE. Dialysis initiation, modality choice, access, and prescription: conclusions from a Kidney Disease: Improving Global Outcomes (KDIGO) Controversies Conference. Kidney Int. 2019;96(1):37-47

38. Freidin N, O'Hare AM, Wong SPY. Person-centered care for older adults with kidney disease: Core Curriculum 2019. Am J Kidney Dis. 2019:11. pii: S0272-6386(19)30170

39. Brown EA, Bargman JM, Li PK. Managing Older Patients on Peritoneal Dialysis. Perit Dial Int. 2015;35(6):609-611

40. Aucella F, Brunori G, Dalmartello M, Leosco D, Paolisso G, Marangella M et al. Assessment of the geriatric competence and perceived needs of Italian nephrologists: An internet survey. J Nephrol. 2016 Jun;29(3):385-39
41. Hall RK, Haines C, Gorbatkin SM, Schlanger L, Shaban H, Schell JO et al. Incorporating geriatric assessment into a nephrology clinic: Preliminary data from two models of care. J Am Geriatr Soc. 2016 Oct;64(10):2154-2158

42. Brown EA, Farrington K. Geriatric assessment in advanced kidney disease. Clin J Am Soc Nephrol. 2019 Jul 5;14(7):1091-1093

43. The POLDER (Pathway for Older Patients Reaching End Stage Renal Disease) initiative. Available at https://www.polderstudie.nl/en/about-polder. Accessed Jul, 2019

\section{Correspondence to:}

Ana Farinha

Nephrology Department, Centro Hospitalar de Setúbal

E-mail: alpfarinha@gmail.com 